EFFETTO DI COLTURE STARTER DI ORIGINE LATTIEROCASEARIA SULLE CARATTERISTICHE MICROBIOLOGICHE, CHIMICHE E SENSORIALI DI SALAMI DI DAINO (DAMA DAMA) PRODOTTI SENZA NITRITI

\title{
EFFECT OF SELECTED DAIRY STARTER CULTURES ON MICROBIO- LOGICAL, CHEMICAL AND SENSORY CHARACTERISTICS OF VENISON (Dama dama) NITRITE-FREE DRY-CURED SAUSAGES
}

\author{
Parmegiani S. ${ }^{1}$, Vizzani A. ${ }^{2}$, Sechi P., Cenci Goga B. ${ }^{2}$ \\ ${ }^{1}$ Master in Sanità Pubblica Veterinaria e Igiene degli Alimenti, Università degli Studi di Perugia \\ ${ }^{2}$ Scuola in Ispezione degli Alimenti di Origine Animale, Università degli Studi di Perugia
}

SUMMARY

KEYWORDS

\begin{abstract}
The aim of this study was the evaluation of the use of selected lactic acid bacteria starter culture of dairy origin in the production of nitrite-free low-acid fermented venison (Dama dama) sausage (Salame di daino) produced in a small-scale plant in Umbria (Italy), and their effect on microbiological, physico-chemical and sensorial properties of the products. Salame di daino was obtained with two different processes: with and without the addition of selected LAB starter cultures. Microbial counts of safety indicators were lower in salami made with the addition of starter cultures. Pathogens after the first week of ripening were only detected from salami made without the addition of starter cultures. Control salami were paler and harder, whereas those made with the addition of starter cultures slightly saltier, juicier and in general more acceptable. Selected dairyorigin starter (SDS) cultures did prevent the growth of safety indicators, greatly reduced the rate of isolation of pathogens and increased the acceptability of fullripened salami.
\end{abstract}

starter culture, dry-cured sausage, Lactococcus lactis ssp. lactis, Lactobacillus casei ssp. casei, fallow deer, Dama dama.

\section{INTRODUZIONE}

Negli ultimi anni si è assistito a un crescente interesse da parte dei consumatori per la carne di selvaggina. La carne di selvaggina ha consistenza e sapore caratteristici: è generalmente più scura, ha un gusto più forte ed è spesso più dura rispetto alla carne proveniente dagli animali d'allevamento. I prodotti di carne di selvaggina, fermentati ed essiccati, sono diventati più popolari e più facilmente reperibili sul mercato (1-4). La loro presenza rimane, tuttavia, molto ristretta e di stagione e la qualità della carne è influenzata dalla disponibilità del pascolo e dall'attività degli animali (5). La selvag- gina e la carne di daino in particolare, oggi sono considerate dai consumatori sane e ottenute con un maggiore rispetto per l'ambiente, se paragonate a quelle di animali allevati (3). Per queste ragioni, i prodotti trasformati a base di carne di daino e selvaggina sono spesso realizzati senza l'aggiunta di nitriti e nitrati (6). Gli ingredienti di base sono la carne magra di daino (28\%), il magro (spalla e addome) di suino (55\%), il grasso di suino $(16 \%)$, sale $(25-30 \mathrm{~g} / \mathrm{Kg})$, pepe $(5$ $\mathrm{g} / \mathrm{kg})$, aglio $(2 \mathrm{~g} / \mathrm{Kg})$, vino bianco $(2 \mathrm{ml} / \mathrm{Kg})$ e acido ascorbico $(2 \mathrm{~g} / \mathrm{Kg})$. Nella produzione sperimentale oggetto di questa nota, non sono stati aggiunti latte in polvere, zuccheri, né nitriti e nitrati. Fondamentalmente, la sicurezza del 
prodotto, così insaccato, si basa sulla bassa temperatura di fermentazione. Una delle sfide più interessanti per l'industria della carne è la produzione di salumi a bassa acidità, senza aggiunta di nitrati e nitriti, preservando le caratteristiche igieniche, il sapore e l'aroma originali. L'associazione di due stipiti di Lactococcus lactis ssp. lactis e uno di Lactobacillus casei spp. casei, originariamente isolati dai formaggi tradizionali, prodotti in caseifici di piccole dimensioni in Umbria, è stata scelta per la sua leggera, ma evidente attività acidificante, anche in assenza di zucchero aggiunto o latte scremato $(7 ; 8)$.

\section{MATERIALI E METODI}

Colture starter. Prima della produzione dei salami, gli stipiti liofilizzati di colture starter sono stati coltivati aerobicamente in Nutrient Broth (NB, CM0001 Oxoid, Basingstoke, UK) a $37^{\circ} \mathrm{C}$ per 24 ore. Ogni stipite è stato coltivato in latte scremato (BD Difco, Franklin Lakes, NJ, USA, 232100) a $37^{\circ} \mathrm{C}$ per 24 ore. La conta totale su Nutrient Agar, (NA, Oxoid CM0003), incubato a $37^{\circ} \mathrm{C}$ in ambiente aerobio per 24 ore è stata di circa 1 x $10^{7} \mathrm{UFC} / \mathrm{ml}$. Produzione di salame $e$ procedura di campionamento - Una volta aggiunti tutti gli ingredienti alla carne macinata, il tutto è stato diviso in due impasti: in uno sono state aggiunte le colture starter, con un rapporto cocchi-bacilli di 2:1, l'altro è stato usato come controllo. Tutti i salami sono stati insaccati in budelli naturali di suino, appesi e conservati in cella condizionata per la fermentazione e stagionatura per 27 giorni, secondo il programma indicato di seguito: tempo/temperatura/umidità relativa: ore $0-5 / 6{ }^{\circ} \mathrm{C} / 65 \% \mathrm{UR}$; ore $5-10 / 12 / 65$; ore $10-15 / 22 / 80$; ore $15-20 / 20 / 85$; ore 20 24/18/90; giorni 1-6/16/90; giorni 7-21/15/85. Il prelievo dei campioni è stato effettuato al giorno 0 (miscela di carne subito dopo l'insacco), al giorno 6 (fine fermentazione), e al giorno 27 (fine stagionatura). Ad ogni tempo di campionamento, sono stati prelevati tre campioni per ciascun salame. Tutte le analisi sono state condotte, in triplo, entro 30 minuti dal campionamento. Analisi microbiologiche - $25 \mathrm{~g}$ di campione sono stati trasferiti asetticamente in $225 \mathrm{ml} \mathrm{di}$ acqua peptonata tamponata sterile, (Oxoid, CM1049) ed omogeneizzati in Stomacher (PBI International) prima dell'allestimento delle diluizioni. Le diluizioni decimali seriali, in acqua peptonata tamponata, sono state inoculate sui terreni di coltura, utilizzando $1 \mathrm{ml}$ per la semina per inclusione per la conta microbica totale, e $0,1 \mathrm{ml}$ per quella di superficie, su agar selettivi. La flora aerobica mesofila totale è stata determinata in Plate Count Agar (Oxoid), incubato a $30^{\circ} \mathrm{C}$ per $72 \mathrm{~h}$; Lactococcus spp. in M17 agar (Oxoid, CM0785) a cui è stato aggiunto il $10 \% \mathrm{v} / \mathrm{v}$ di lattosio incubato a $37{ }^{\circ} \mathrm{C}$ per 48 ore, Lactobacillus spp. su de Man, Rogosa, Sharpe (MRS) Agar (Oxoid) acidificato a pH 5.5, incubato a $30^{\circ} \mathrm{C}$ per 72 ore in condizioni anaerobiche (Gas generating kit, Oxoid, BR0038), enterococchi su Slanetz e Bartley (SB) Agar (Oxoid, CM0377), incubato a $37^{\circ} \mathrm{C}$ per 48 h; Enterobacteriaceae, su Violet Red Bile Glucose Agar (VRBG) (Oxoid, CM0485), incubato a 37 ${ }^{\circ} \mathrm{C}$ per $24 \mathrm{~h}$; organismi coliformi su Violet Red Bile Lactose Agar (VRBL) (Oxoid, CM0107), incubato a $30^{\circ} \mathrm{C}$ per $24 \mathrm{~h}$, Micrococcaceae su Mannitol Salt Agar (Oxoid, CM0085), incubato a $37^{\circ} \mathrm{C}$ per 48 h. I presunti $S$. aureus sono stati determinati mediante semina su Tryptose blood agar base, (Oxoid, CM0233 con l'aggiunta di sangue bovino): tutte le colonie mucose, di colore bianco-grigiastro o giallo oro, di diametro da 3 a $5 \mathrm{~mm}$, con una zona distinta di emolisi incompleta, sono state selezionate, e quindi seminate su Rabbit Plasma Fibrinogen (RFP) Agar (Oxoid, CM0961, con Supplemento RFP, Oxoid, SR0122), e incubate a $37{ }^{\circ} \mathrm{C}$ per 48 h. Dopo l'incubazione sono state esaminate microscopicamente, dopo colorazione di Gram, tipiche colonie nere, convesse con un alone opaco. Inoltre, è stata ricercata la presenza di Clostridium spp., Salmonella spp. e Listeria spp. La presenza di clostridi solfito-riduttori è stata determinata mediante Perfringens Agar (Oxoid, CM0543) a cui sono stati aggiunti i Supplementi Perfringens A (Oxoid, SR0076) e B (Oxoid, SR0077). È stato usato il metodo ISO 6579 (ISO, 2007) per isolare Salmonella spp. (9). È stato utilizzato il metodo ISO 11290 (ISO, 1996) per isolare Listeria monocytogenes (10). Pertanto, le piastre con meno di 30 UFC non sono state utilizzate per i dati delle analisi, e quando questo è stato applicato alla più bassa diluizione, $\mathrm{i}$ risultati sono stati registrati come $<300$ per la semina di inclusione e $<3.000$ per la semina di superficie. I campioni con almeno una tipica colonia (dopo un'ulteriore identificazione per S.aureus) nella più bassa diluizione, sono stati registrati come positivi, indipendentemente dal numero delle colonie. Analisi fisico-chimiche_aw, pH e umidità sono stati determinati durante la stagionatura, mentre la composizione chimica e le ceneri nel giorno dell'insacco e a fine stagionatura. L'umidità, la composizione chimica e le ceneri sono stati determinati in base ai metodi AOAC (AOAC, 2000) (11). Valutazione sensoriale - Panel test su entrambi i salami. Analisi dei risultati - Ad ogni tempo di campionamento è stata calcolata la media aritmetica dei tre campioni di ciascun salame, tutti i valori (convertiti in log per le analisi microbiologiche) sono stati analizzati con GraphPad Instat, v. 3.0b per Mac OS X 
ed i grafici sono stati ottenuti con GraphPad Prism v. 5.0d per Mac OS X.

\section{RISULTATI E CONCLUSIONI}

\author{
Analisi microbiologica - Flora aerobica mesofila \\ totale e $L A B$
}

Il giorno dell'insaccamento, i salami di controllo non contenevano batteri lattici, mentre i salami con l'aggiunta di colture SDS contenevano più di $10^{7} \mathrm{UFC} / \mathrm{g}$ di lactococchi e lactobacilli. Per i salami di controllo, la conta della flora aerobica mesofila totale, ha dato come log di UFC/g (media aritmetica), un range che variava da 5,66 a 6,00 , mentre in quelli realizzati con l'aggiunta di colture SDS il range variava da 7,45 a 8,47. Nei salami fatti con l'aggiunta delle colture SDS, le conte dei lactococchi variavano da 7,32 a 7,69, mentre le conte di Lactobacillus spp. variavano da 7,00 a 7,30. Al termine della fermentazione (giorno-6), le conte dei batteri lattici avevano raggiunto più di $10^{8} \mathrm{UFC} / \mathrm{g}$ nei salami fatti con l'aggiunta di colture SDS (da 8,81 a 8,98 per i lactococchi e da 8,00 a 8,62 per i lactobacilli) e più di $10^{7} \mathrm{UFC} / \mathrm{g}$ in quelli di controllo (da 8,00 a 8,28 per i lactococchi e da 7,00 a 8,08 per i lactobacilli). Le conte della flora aerobica mesofila totale erano di conseguenza simili (da 8,86 a 8,93 nei salami con l'aggiunta di colture SDS e da 8,00 a 8,31 nei salami di controllo). Al termine della stagionatura (giorno-27), i lactococchi e i lactobacilli erano superiori a $10^{7}$ UFC/g sia nei salami fatti con l'aggiunta di colture SDS (lactococchi da 7,76 a 8,81 e lactobacilli da 7,52 a 8,16 ) sia nei salami di controllo (lactococchi da 7,50 a 7,59 e lactobacilli da 7,81 a 7,98). Le conte della flora aerobica mesofila totale erano simili alle conte dei lactococchi (da 7,89 a 8,67 nei salami di controllo e da 8,21 a 8,810 nei salami con l'aggiunta di colture SDS).

Enterococchi, micrococchi, enterobatteri e coliformi

Il giorno dell'insaccamento, la media delle conte degli enterococchi era inferiore a $10^{5} \mathrm{UFC} / \mathrm{g}$ nei salami di controllo e superiore a $10^{5} \mathrm{UFC} / \mathrm{g}$ nei salami fatti con l'aggiunta di colture SDS ed i valori variavano da 4,00 a 4,60 e da 5,34 a 5,98 , rispettivamente. La media delle conte delle Enterobacteriaceae era compresa tra $10^{4}$ e $10^{5}$ UFC/g in entrambi i tipi di salame ed i valori variavano da 5,11 a 5,30 in quelli di controllo e, da 4,30 a 5,48 , in quelli con l'aggiunta di colture SDS. I valori medi dei coliformi erano oltre $10^{4}$ UFC/g in entrambi i gruppi (da 4,70 a 4,90 nei salami di controllo e da 4,95 a 5,70 nei salami fatti con l'aggiunta di colture SDS). La media delle conte delle Micrococcaceae era compresa tra $10^{4}$ e $10^{5} \mathrm{UFC} / \mathrm{g}$ in entrambi i tipi di salame ed i valori variavano da 4,00 a 4,60 nei salami di controllo e da 5,48 a 5,80, nei salami fatti con l'aggiunta di colture SDS. Al termine della fermentazione (giorno-6), gli enterococchi avevano raggiunto valori medi di oltre $10^{5} \mathrm{UFC} / \mathrm{g}$, sia nei salami fatti con l'aggiunta di colture SDS (da 5,88 a 5,97 ) sia nei salami di controllo (da 5,00 a 5,15). I valori medi delle Enterobacteriaceae erano oltre $10^{5} \mathrm{UFC} / \mathrm{g}$ nei salami di controllo (da 4,89 a 5,02 ) e inferiore a $10^{5} \mathrm{UFC} / g$ nei salami fatti con l'aggiunta di colture SDS (da 4,86 a 5,00). I coliformi erano oltre $10^{4} \mathrm{UFC} / \mathrm{g}$ nei salami controllo (da 3,90 a 5,75) e sotto $10^{4} \mathrm{UFC} / \mathrm{g}$ nei salami fatti con l'aggiunta di colture SDS (da 3,2 a 3,95). Le Micrococcaceae avevano raggiunto valori medi di oltre $10^{5} \mathrm{UFC} / \mathrm{g}$ in entrambi i tipi di salame con conte che variavano da 5,60 a 6,14 nei salami di controllo e da 6,00 a 6,32 nei salami fatti con l'aggiunta di colture SDS. Al termine della stagionatura (giorno-27), i valori medi degli enterococchi erano $10^{5} \mathrm{UFC} / \mathrm{g}$ in entrambi i tipi di salame (da 5,05 a 6,56 nei salami fatti con l'aggiunta di colture SDS e da 4,60 a 6,00 nei salami di controllo). I valori medi delle Enterobacteriaceae erano oltre $10^{4}$ UFC/g nei salami di controllo (da 4,27 a 4,67) e in quelli fatti con l'aggiunta di colture SDS (da 3,82 a 4,50 ). I coliformi non sono stati rilevati in entrambi i tipi di salami. Le Micrococcaceae hanno raggiunto valori medi di oltre $10^{5} \mathrm{UFC} / \mathrm{g}$ in entrambi i tipi di salame, con le conte che variano da 4,92 a 5,57 nei salami di controllo e da 5,49 a 5,51 nei salami fatti con l'aggiunta di colture SDS.

Patogeni

$S$. aureus è stato isolato solo nei salami di controllo e solo nel giorno dell'insaccamento. Gli anaerobi sporigeni, non sono mai stati rilevati. Salmonella spp. e Listeria spp. sono state rilevate nei salami di controllo fino alla fine della fermentazione (giorno-6), mentre negli altri, fatti con l'aggiunta di colture SDS, solo nel giorno dell'insaccamento.

Analisi fisico-chimiche

I valori medi di aw, nel giorno del riempimento erano al di sopra di 0.96 per entrambi gli impasti (da 0,96 a 0,97 in entrambi i tipi di salame) e hanno raggiunto valori di 0,94 (da 0,94 a 0,95 in entrambi i tipi) e 0,92 nei salami realizzati con l'aggiunta di colture SDS, alla fine della fermentazione (giorno-6), ed i valori di 0,83 (da 0,83 a 0,84 sia nei salami di controllo, sia nei salami con l'aggiunta di colture SDS) al termine della stagionatura (giorno-27). Nel giorno dell'insaccamento, i valori medi di umidità erano al di sopra del $62 \%$ per entrambi gli impasti $(62,89 \%$ nei campioni di controllo e $62,87 \%$ nei salami con l'aggiunta di colture SDS), mentre alla fine della fermentazione (giorno-6) erano superiori al 50\% (54,90\% e 59,60\% nei salami di 
controllo e nei salami fatti con l'aggiunta di colture SDS, rispettivamente). Al termine della stagionatura (giorno-27), i valori medi erano più del $28 \%(28,93 \%$ nei salami di controllo e $30,82 \%$ per i salami fatti con l'aggiunta di colture SDS). I valori medi di $\mathrm{pH}$, nel giorno dell'insaccamento erano superiori a 6,50 in entrambi gli impasti (da 6,36 a 6,66 nei salami di controllo, e da 6,45 a 6,65 nei salami con l'aggiunta di colture SDS) e hanno raggiunto i valori di 6,24 nei salami di controllo (da 6,12 a 6,32) e 5,64 nei salami fatti con l'aggiunta di colture SDS alla fine della fermentazione (giorno-6) (da $5,55$ a 5,76$)$ e valori di 7,01 nei salami di controllo (da 6,67 a 7,22) e 7,00 nei salami fatti con l'aggiunta di colture SDS alla fine della stagionatura (giorno-27) (da 6,98 a 7,04). Il giorno del riempimento i valori medi di proteine, espressi in 100/g di sostanza secca, erano 47,28 (da 45,98 a 48,53) nei salami di controllo e di 50,51 (da 50,08 a 51,36) nei salami con l'aggiunta di colture SDS; il grasso, espresso in 100/g di sostanza secca, era 44,07 (da 43,11 a 45,19) e 40,98 (da 40,50 a 41,90 ), rispettivamente. I valori medi per le ceneri espressi in 100/g di sostanza secca, erano 8,66 nei salami di controllo (da 8,40 a 8,65 ) e 8,51 (da 8,01 a 8,95 ) nei salami con l'aggiunta di colture SDS. Le analisi condotte nei salami a completa stagionatura (giorno 27) hanno prodotto dati simili.

Valutazione sensoriale

L'uniformità di colore variava da 4,17 nei salami di controllo a 5,17 in quelli fatti con l'aggiunta di colture SDS; l'intensità di colore variava da 4,83 nei salami di controllo a 5,00 nei salami fatti con l'aggiunta di colture SDS; la connessione grasso/magro da 4,50 a 5,00, e la distribuzione grasso/magro da 5,33 a 4,83, rispettivamente. Per quanto riguarda il gusto, l'odore e l'aroma, l'acidità variava da 3,50 nei salami di controllo a 2,33 nei salami fatti con l'aggiunta di colture SDS; l'irrancidimento da 3,33 a 1,50; l'amarezza da 3,17 a 2,33; il sapore salato da 3,67 a 5,17 ; il sapore di muffa da 2,50 a 1,83 ; il gusto piccante da 3,33 a 3,83 e l'intensità di sapore da 5,50 a 6,00, rispettivamente. Per quanto riguarda gli attributi di consistenza, l'elasticità era di 3,33 in entrambi i tipi di salame; la durezza variava da 3,33 nei salami di controllo a 4,5 nei salami con l'aggiunta di colture SDS; la coesione da 3,50 a 5,17, rispettivamente; la masticabilità da 5,50 a 4,83; la succosità da 4,00 a 4,17 ; il grasso da 4,33 a 3,83 , rispettivamente. L'accettabilità complessiva variava da 5,50 nei salami di controllo a 5,67 nei salami realizzati con l'aggiunta di colture SDS.

L'aggiunta di colture starter nei salami di daino ha impedito la crescita degli indicatori di sicurezza, ha determinato una considerevole ridu- zione degli agenti patogeni e dei livelli di Enterobacteriaceae e soprattutto di coliformi. Ha migliorato leggermente le caratteristiche sensoriali a fine stagionatura, aumentandone l'accettabilità, ed evitando l'uso di nitriti e nitrati. Non ha inibito la crescita degli enterococchi e delle Micrococcaceae, i quali sono in grado di modulare l'aroma dei prodotti (12-15). I salami senza l'aggiunta di colture starter sono risultati essere più rancidi e con un più forte sapore di muffa, mentre quelli con l'aggiunta di colture starter sono leggermente più salati, più coesi e più accettabili.

\section{RINGRAZIAMENTI}

Ricerca condotta con finanziamento Fondazione Cassa di Risparmio di Perugia, Progetto: 2006.0258.020 - Sviluppo locale.

\section{BIBLIOGRAFIA}

1. Bertolini, R., Zgrablic, G., \& Cuffolo, E. (2005). Wild Game Meat: Products, Market, Legislation and Processing Controls. Veterinary Research Communications, 29(2), 97-100.

2. Garcİa-Riuz, A., Mariscal, C., \& Soriano, A. (2007). Influence of hunting-season stage and ripening conditions on nitrogen fractions and degradation of myofibrillar proteins in venison (Cervus elaphus) chorizo sausages. Meat Science, 76(1), 74-85.

3. Hoffman, L. C., \& Wiklund, E. (2006). Game and venison's meat for the modern consumer. Meat Science, 74(1), 197-208.

4. Severini, M., Stocchi, R., Cenci-Goga, B., \& Scorciarini-Coppola, A. (1999). Caratteristiche qualitative e microbiologiche di salami di bufalo. In IX Convegno Nazionale Associazione Italiana Veterinari Igienisti (pp. 201-205). Colle Val d'Elsa.

5. Anonymous. (2011). Fallow deer. Forestry Commission, UK, access date: February 2011 http://www.forestry.gov.uk/forestry/Fallowd eer.

6. Zarringhalami, S., Sahari, M. A., \& HamidiEsfehani, Z. (2009). Partial replacement of nitrite by annatto as a colour additive in sausage. Meat Science, 81(1), 281-284.

7. Cenci-Goga, B., Ranucci, D., Miraglia, D., \& Cioffi, A. (2008). Use of starter cultures of dairy origin in the production of Salame nostrano, an Italian dry-cured sausage. Meat Science, 78(4), 381-390.

8. Clementi, F., Cenci-Goga, B., TrabalzaMarinucci, M., \& Di Antonio, E. (1998). Use of selected starter cultures in the 
production of farm manufactured goat cheese from thermized milk. Italian Journal of Food Science, 10(1), 41-56.

9. ISO. (2007). Microbiology of food and animal feeding stuffs -- Horizontal method for the detection of Salmonella spp. ISO 6579:2002/Amd 1:2007. International Organization for Standardization, Geneva, Switzerland.

10. ISO. (1996). Microbiology of food and animal feeding stuff. Horizontal method for detection and enumeration of Listeria monocytogenes. Detection Method. ISO 11290-1:1996. International Organization for Standardization, Geneva, Switzerland.

11. AOAC. (2000). Official Methods of Analysis of AOAC International (OMA), 17th Edition (17 ed.). Wasington, DC, USA: Association of Official Analytical Chemists.

12. Corbiere Morot-Bizot, S., Leroy, S., \& Talon, R. (2007). Monitoring of staphylococcal starters in two French processing plants manufacturing dry fermented sausages. Journal of Applied Microbiology, 102(1), 238-244.

13. García Fontán, M. C., Lorenzo, J. M., Parada, A., Franco, I., \& Carballo, J. (2007). Microbiological characteristics of "androlla", a Spanish traditional pork sausage. Food Microbiology, 24(1), 52-58.

14. González-Fernández, C., Santos, E. M., Rovira, J., \& Jaime, I. (2006). The effect of sugar concentration and starter culture on instrumental and sensory textural properties of chorizo-Spanish dry-cured sausage. Meat Science, 74(3), 467-475.

15. Leroy, F., Verluyten, J., \& De Vuyst, L. (2006). Functional meat starter cultures for improved sausage fermentation. International Journal of Food Microbiology, 106(3), 270-285. 